\title{
Modelo de toma de decisiones de mantenimiento basado en la predicción de vida útil para componentes de sistemas eólicos en Costa Rica
}

\section{Maintenance decision making model based on useful life prediction for wind power generation systems components installed in Costa Rica}

Ana Laura Loría-García1', Edgardo Mateo Villalobos-Granados², Carlos Piedra-Santamaría ${ }^{3}$,

Loría-García, A; Villalobos-Granados, E; Piedra-Santamaría, C . Modelo de toma de decisiones de mantenimiento basado en la predicción de vida útil para componentes de sistemas eólicos en Costa Rica. Tecnología en Marcha. Vol. 30-3. Julio-Setiembre 2017. Pág 129-141.

DOI: $10.18845 / \mathrm{tm} . v 30 i 3.3279$ ta Rica. Costa Rica. Correo electrónico: analoga2992@gmail.com.

2 Costarricense. Ingeniero en Mantenimiento Industrial. Instituto Tecnológico de Costa Rica. Costa Rica. Correo electrónico: edgatolobo@gmail.com.

3 Costarricense. Ingeniero en Mantenimiento Industrial. Instituto Tecnológico de Costa Rica. Costa Rica. Correo electrónico: cpiedra@itcr.ac.cr. 


\title{
Palabras clave
}

Confiabilidad; Turbinas Eólicas; Redes Neuronales Artificiales; Optimización de Costos.

\section{Resumen}

Este estudio presenta un modelo de toma de decisiones de mantenimiento, donde se aplica el monitoreo de condición a componentes rotativos de aerogeneradores de eje horizontal instalados en Costa Rica, reduciendo la incidencia de fallas inesperadas, lo cual es consecuencia de la política de dejar fallar o apegarse a las recomendaciones del fabricante sin tomar en cuenta el entorno operativo, prácticas comunes en la industria eólica del país.

Se ofrece un modelo donde se definen dos valores umbrales de probabilidad de falla, los cuales se utilizan en la toma de decisiones de reemplazo de componentes, con el fin de optimizar los costos de operación y mantenimiento. Además, se brindan las pautas iniciales para ejecutar esta estrategia de mantenimiento en un proyecto eólico.

Las predicciones de los porcentajes de vida requeridas por el modelo ofrecido, se obtienen utilizando redes neuronales artificiales, las cuales tienen como entradas variables de condición representativas para cada componente en estudio (rotor, rodamiento principal, caja multiplicadora y generador eléctrico).

\section{Keywords}

Reliability; Wind Turbines; Artificial Neural Networks; Cost Optimization.

\begin{abstract}
This study covers a maintenance decision making model, where condition monitoring is applied to rotating components of horizontal axis wind power generation systems installed in Costa Rica, in order to reduce the incidence of unexpected failures, which are consequences of the run-to-failure policy or following strictly the manufacturers' suggestions without considering the operational environment, very common practices in the national wind industry.

Aiming the optimization of operation and maintenance costs, a model where two failure probability threshold values are defined is presented. These threshold values allow the component replacement decision making. Moreover, the initial guidelines for executing this strategy in a wind farm are offered in this paper.

The life percentage predictions required by the offered model, are obtained using artificial neural networks for each component (rotor, main bearing, gearbox and electric generator), which use representative condition monitoring variables as inputs.
\end{abstract}

\section{Introducción}

La mayoría de los sistemas electromecánicos modernos son grandes, complejos, con características especiales y estructuras particulares [1]. Cuando un sistema complejo falla, las consecuencias pueden ser dramáticas; por ejemplo, pueden ocurrir pérdidas económicas sensibles, afectación de la salud y bienestar de seres humanos y daños serios al ambiente [2], [3]. 
Para mitigar estos efectos, el mantenimiento industrial ha pasado de ser un asunto meramente técnico a uno de administración estratégica. Este nuevo rol implica brindar soluciones a problemas cada vez más complejos, como la predicción oportuna de fallas y la gestión eficiente de activos.

Los costos de operación y mantenimiento (O\&M) en la industria eólica son altamente representativos, según se muestra en el cuadro 1, por lo tanto, reducirlos es un factor clave para la comercialización de la energía eólica [4], [5]. Una herramienta útil para lograr este objetivo es la aplicación de modelos estadísticos y probabilísticos para evaluar la confiabilidad de un sistema con base en la de sus componentes, su diseño y la disposición de los mismos en el sistema [6], [1]. Estos modelos tienen un alto nivel de aplicación en las industrias más exigentes a nivel mundial, como la de energías renovables.

Costa Rica se ha propuesto ser carbono neutral para el año 2100 [7], lo cual implica, entre otros aspectos, producir el 100\% de su electricidad con energías renovables, situación que ha llevado al crecimiento acelerado de la energía eólica, duplicándose la capacidad instalada entre 2010 y 2015 [8].

A pesar del crecimiento de la industria eólica, aún existe un rezago en estrategias de mantenimiento propias de esta área, situación inaceptable debido a que la energía a partir del viento es de naturaleza intermitente y difusa [9]. Además de la limitada accesibilidad para realizar acciones de mantenimiento y alta criticidad de la mayoría de los componentes de los aerogeneradores, las turbinas eólicas se encuentran sujetas a cargas mecánicas irregulares [10], por lo tanto, es imprescindible garantizar disponibilidad y una alta confiabilidad durante los períodos de viento con velocidades dentro del rango de producción.

Es fundamental contar con un modelo de gestión de mantenimiento que considere el deterioro de los aerogeneradores y por lo tanto sea basado en la condición, con el fin de lograr una significativa reducción en los costos de O\&M. Lo anterior permite que recursos como tiempo y presupuesto sean asignados de manera óptima.

Tomando en cuenta los factores mencionados, se busca generar una estrategia alternativa a las políticas estrictamente correctivas y de reemplazo en intervalos constantes, utilizando el monitoreo de condición para evaluar el deterioro progresivo y ejecutar reemplazos únicamente cuando es necesario, y antes de que ocurran fallas catastróficas, lo cual asegura el aprovechamiento de la vida útil de los distintos componentes.

Cuadro 1. Costos de explotación generales para proyectos eólicos.

\begin{tabular}{|c|c|c|c|c|}
\hline Rubro & $\begin{array}{c}\text { Operación y } \\
\text { mantenimiento }\end{array}$ & Terrenos & $\begin{array}{c}\text { Seguros e } \\
\text { impuestos }\end{array}$ & Gestión y administración \\
\hline Costo (\%) & 57 & 16 & 14 & 13 \\
\hline
\end{tabular}

Fuente: Global Wind Energy Council, 2016.

\section{Estado de la generación eólica en Costa Rica}

En Costa Rica, no se cuenta con datos históricos de fallas y tiempos de paro de los aerogeneradores [11], [12], por lo tanto, se debe comenzar con una recolección piloto por un año, aunque se recomienda la toma de datos por un período de al menos $20 \%$ de la vida útil 
total de diseño del sistema [13]. Este lapso sería de cuatro años, ya que en general, la vida útil de diseño de los aerogeneradores es de 20 años, según lo indica la norma IEC 61400-1 [14].

Al no existir estos datos en el entorno operacional de Costa Rica, se toman como referencia algunas bases de datos existentes [13], [15], [16], [17], donde se observa que los componentes rotativos que presentan mayores tiempos de paro son la caja multiplicadora, el generador eléctrico, el rodamiento principal y el rotor.

En el cuadro 2, se observa la distribución de la capacidad instalada al 2016, la cual representa un $9,07 \%$ del Sistema Eléctrico Nacional.

Cuadro 2. Plantas de generación eólica en Costa Rica y modelos de los distintos aerogeneradores.

\begin{tabular}{|c|c|c|c|c|c|c|}
\hline Empresa & Planta & Unidades & $\begin{array}{l}\mathrm{kW} \text { de } \\
\text { placa }\end{array}$ & Fabricante & Modelo & $\begin{array}{c}\text { Caja } \\
\text { multiplicadora }\end{array}$ \\
\hline \multirow[t]{2}{*}{ ICE } & Tejona & 30 & 18480 & Vestas & V42/660 & Sí \\
\hline & P.E Guanacaste & 55 & 49700 & Enercon & E44/900 & No \\
\hline \multirow[t]{4}{*}{ BOT } & Chiripa & 33 & 49500 & Acciona & AW77/1500 & Sí \\
\hline & Orosí & 25 & 50000 & Gamesa & G87/2000 & Sí \\
\hline & $\begin{array}{c}\text { Plantas Eólicas } \\
\text { S.R.L }\end{array}$ & 55 & 22660 & Kenetech & $33 \mathrm{M}-\mathrm{VS}$ & Sí \\
\hline & Aeroenergía & 9 & 6750 & Neg Micon & NM48/750 & Sí \\
\hline \multirow[t]{3}{*}{ Privado } & Tilawind & 7 & 21000 & Vestas & V90/3000 & Sí \\
\hline & Movasa & 32 & 20000 & Neg Micon & NM48/750 & Sí \\
\hline & Vientos del Este & 4 & 12000 & Wobben & E82/3000 & No \\
\hline C.N.F.L & Valle Central & 17 & 15300 & Enercon & E44/900 & No \\
\hline Coopesantos & Los Santos & 15 & 12750 & Gamesa & G52/850 & Sí \\
\hline \multicolumn{2}{|c|}{ Total Eólico } & 282 & 278140 & & & \\
\hline
\end{tabular}

Fuente: Instituto Costarricense de Electricidad, 2016 [18]

Los casos de un proyecto eólico en el cual se generaron pérdidas de \$2,1 millones por bajos niveles de producción [19] y otro parque que detuvo operaciones en 2013 por falta de rentabilidad [20], son situaciones que evidencian la necesidad de contar con una estrategia de gestión que permita reducir los costos de O\&M de los parques eólicos del país, y que además se adapte a las características técnicas y entorno operacional de cada proyecto eólico.

\section{Configuración del sistema y reglas de operación}

Por definición, un componente es la mínima unidad que constituye un sistema, el cual se estudia de forma aislada [21]. En un sistema, los componentes interactúan entre sí para llevar a cabo funciones operacionales específicas [22], por lo tanto, la condición del sistema en general, depende de la condición de sus componentes [1]. Es importante entonces conocer la configuración del sistema para comprender el comportamiento del mismo ante las fallas. 


\section{Configuración del sistema}

La configuración del sistema describe la manera en la cual el sistema se encuentra conectado, así como sus reglas de operación [22]. Para evaluar la confiabilidad de sistemas, estos suelen descomponerse jerárquicamente de acuerdo con el desempeño de los componentes.

En un sistema conformado por $n$ componentes conectados en serie, la confiabilidad $\left(R_{\text {Sistema Serie }}\right)$ se calcula como el producto de las confiabilidades individuales $R_{\mathrm{i}}$ [6], tal y como se muestra en la ecuación 1.

$$
R_{\text {Sistema Serie }}=\prod_{i=1}^{n} R_{i}
$$

Por otro lado, para una configuración con $n$ componentes en paralelo, la confiabilidad ( $R_{\text {Sistema }}$ Paralelo) se calcula según la ecuación 2, presentada en [1].

$$
R_{\text {Sistema Paralelo }}=1-\prod_{i=1}^{n}\left(1-R_{i}\right)
$$

Para esquematizar la configuración de los sistemas, se utilizan los diagramas de bloque de confiabilidad (figura 1), donde se indica la relación lógica entre el funcionamiento del sistema y sus componentes [1].

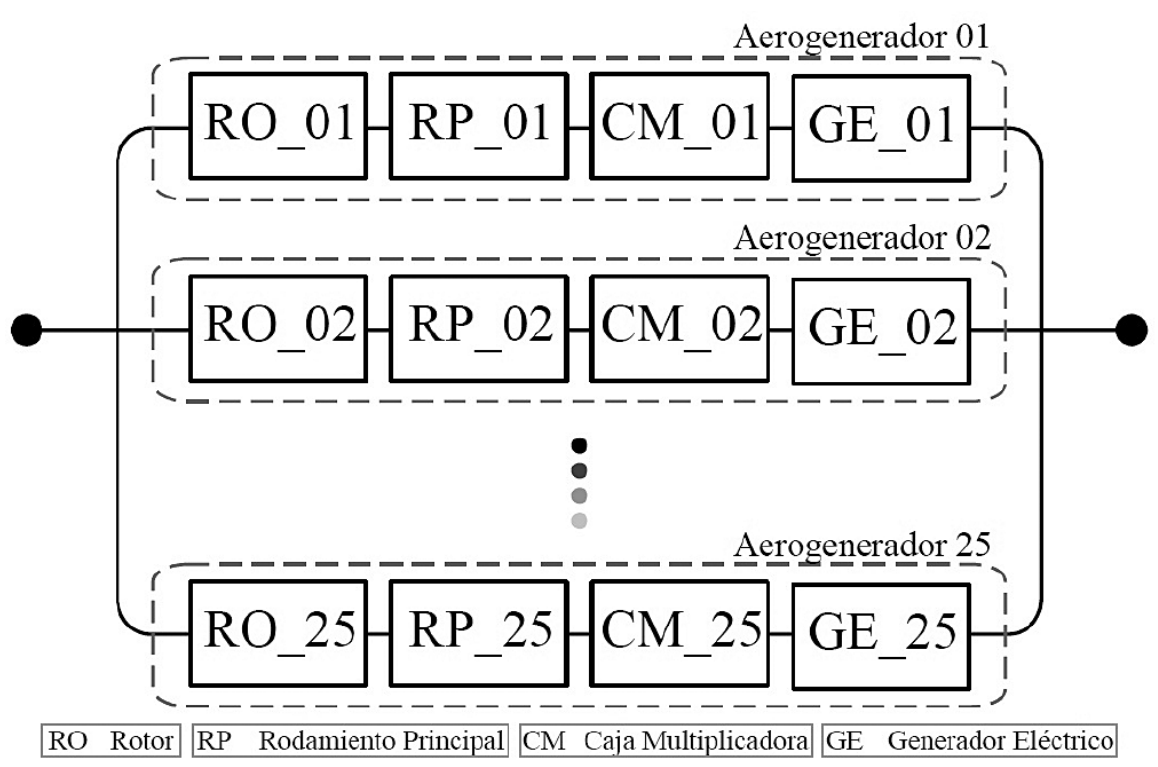

Figura 1. Diagrama de bloques de confiabilidad para un parque eólico de 25 turbinas.

\section{Edad de los componentes}

El concepto de edad calendario de un sistema se refiere al tiempo transcurrido desde que el mismo inició operaciones [22], sin embargo, existe el concepto de edad efectiva, donde se incluye la condición del sistema para conocer el porcentaje de vida útil acumulada en un tiempo específico. Esta edad refleja el efecto del envejecimiento de un componente o sistema a través del tiempo y el rejuvenecimiento luego de distintas intervenciones de mantenimiento [23]. 


\section{Descripción del modelo}

Con el fin de predecir los porcentajes de vida de los distintos componentes, se recurre a las redes neuronales artificiales (RNA), las cuales son modelos matemáticos inspirados en el funcionamiento de las estructuras neurobiológicas humanas [24].

Las neuronas trabajan en forma conjunta para comunicar señales a través de sinapsis entre cuerpos celulares, mismas que se propagan por canales conocidos como axones [25]. Con base en lo anterior, fueron creadas las RNA, correspondientes a "una estructura compuesta de un número de unidades interconectadas, donde cada unidad posee una característica de entrada y salida e implementa una función o computación local" [26]. Los elementos de una RNA se observan en la figura 2.

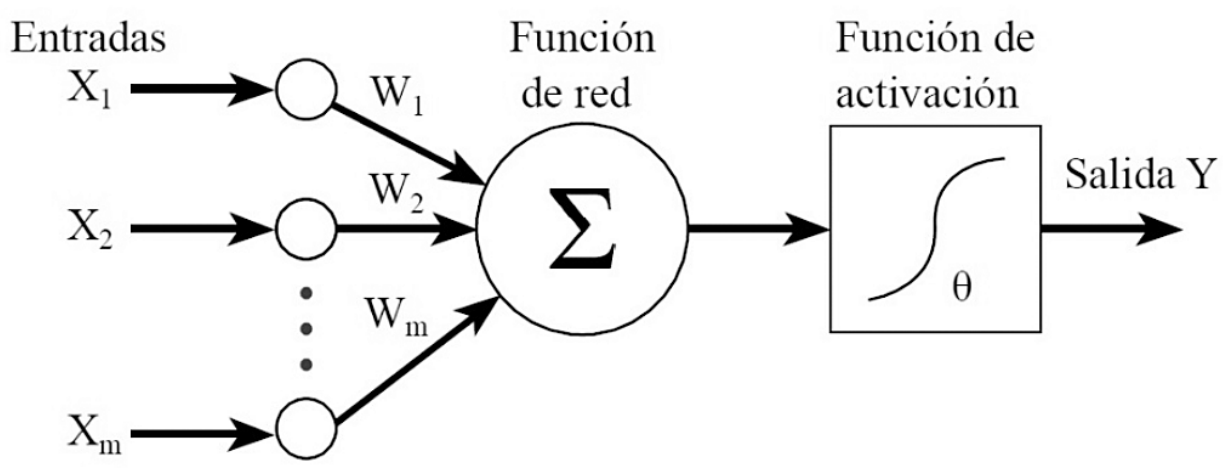

Figura 2. Elementos de una red neuronal artificial.

Se conoce como función de red o propagación, a la suma ponderada ejecutada por cada nodo sobre sus valores de entrada [27]. Posteriormente, existe una función de activación ( $\theta)$, responsable de aproximar las no linealidades del fenómeno [28].

La salida tiene un valor único y puede ser a la vez entrada de una unidad posterior, dependiendo de la configuración de la red.

Con el fin de obtener la salida esperada para las entradas específicas de toda RNA, los pesos de ponderación $(W$ ) deben ajustarse. Este procedimiento de ajuste de pesos se conoce con el nombre de aprendizaje o entrenamiento de la red, y normalmente se realiza con sistemas de computación, utilizando un conjunto de datos conocidos, para minimizar el error entre la salida que se obtiene a través de la red y la salida esperada [29]. Finalmente se lleva a cabo la fase de validación, donde se introducen datos distintos a los de la fase de aprendizaje, para así verificar la capacidad de generalización de la red [30].

En este estudio, se utiliza el perceptrón multicapa, un tipo de RNA donde aparecen capas ocultas entre las de entrada y salida. Esta arquitectura es la más utilizada de todas [31] y ha demostrado brindar resultados satisfactorios en cuanto a predicción de porcentajes de vida de componentes [32].

\section{Predicción de porcentajes de vida}

En cada tiempo de inspección ( $t$ ), se recolectan las mediciones del monitoreo de condición de la máquina y utilizando RNA, es posible estimar el valor del tiempo de falla [33]. En la figura 3, se observa la estructura base de los perceptrones multicapa propuestos para la predicción. 


\section{Capa de entrada Capas ocultas Capa de salida}

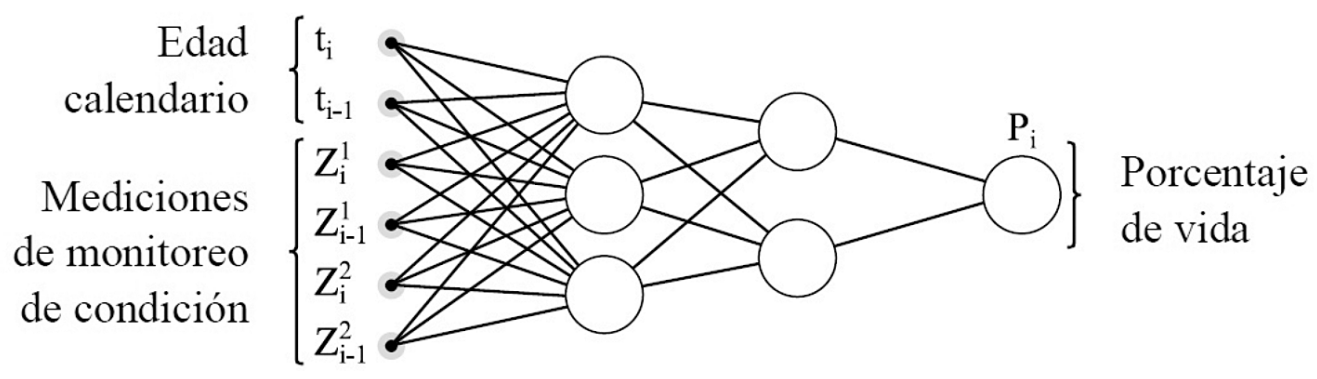

Figura 3. Estructura base de un perceptrón multicapa para predicción de porcentaje de vida.

Las entradas del perceptrón multicapa se insertan en sus valores actual $(i)$ y anterior ( $i-1)$, con el fin de reflejar el cambio en la condición. Existen para este caso dos mediciones de monitoreo de condición, expresadas con la letra $Z$.

La salida del perceptrón multicapa es el porcentaje de vida $\left(P_{j}\right)$ en el tiempo de inspección actual, correspondiente a la edad efectiva. Como ejemplo, si el $P_{i}$ del tiempo de inspección correspondiente a una edad calendario de 210 días es $42 \%$, el tiempo de falla del componente se calcula según la ecuación 3.

$$
\text { Tiempo de falla del componente }=\frac{t_{i}}{P_{i}}=\frac{210}{0,42}=500 \text { días }
$$

Del resultado presentado en la ecuación 3 , se puede interpretar que el tiempo de vida útil remanente será de 290 días.

Durante el entrenamiento y validación de la RNA, se obtienen la media $\left(\mu_{p}\right)$ y la desviación estándar $\left(\sigma_{p}\right)$ de los errores de predicción de porcentaje de vida [34]. Estos valores se utilizan para construir la distribución del tiempo de falla previsto en un determinado punto de inspección. Dados los valores $t_{i}$ y $P_{i}$, el tiempo previsto de falla $T_{p}$ en el punto actual de inspección sigue la distribución normal, como se muestra en la ecuación 4, donde $\mu$ es la media de la distribución de tiempo previsto de falla y la desviación estándar de la distribución de tiempo previsto de falla se denota como $\sigma$.

$$
T_{p} \sim N(\mu, \sigma)=N\left(t_{i} /\left(P_{i}-\mu_{p}\right), \sigma_{p} \cdot t_{i} /\left(P_{i}-\mu_{p}\right)\right)
$$

Estimación de la probabilidad de falla de los componentes y el sistema

La probabilidad de falla $\left(\operatorname{Pr}_{n, m}\right)$ de un componente $m$ de la turbina $n$ en un parque eólico, se define como lo muestra la ecuación 5, presentada en [35].

$$
\operatorname{Pr}_{n, m}=\frac{\int_{t}^{t+L} \frac{1}{\sigma \sqrt{2 \pi}} e^{-0,5\left(\frac{x-\mu}{\sigma}\right)^{2}} d x}{\int_{t}^{\infty} \frac{1}{\sigma \sqrt{2 \pi}} e^{-0,5\left(\frac{x-\mu}{\sigma}\right)^{2}} d x}
$$

Donde la edad del componente en el punto actual de inspección es $t$, mientras que el tiempo de logística se denota con la letra $L$, correspondiente al intervalo entre la decisión de mantenimiento 
y la ejecución del mantenimiento sobre el componente, donde se incluyen eventos propios del desarrollo de las acciones de reemplazo [34]. Se asume que $L$ es igual para todas las acciones de mantenimiento en todos los componentes, con el propósito de simplificar el cálculo.

La probabilidad de falla para la turbina eólica $n\left(\operatorname{Pr}_{n}\right)$, puede expresarse como se muestra en la ecuación 6, al ser este un sistema en paralelo.

$$
P r_{n}=1-\prod_{m=1}^{M}\left(1-P r_{n, m}\right)
$$

Adicionalmente, la confiabilidad del sistema se calcula según lo indicado en la ecuación 7, tal y como se presentó en secciones anteriores.

$$
R_{n}=1-P r_{n}
$$

Estrategia planteada para la toma de decisiones de mantenimiento

Según esta estrategia, se deben determinar dos valores umbrales de probabilidad de falla para definir la política de mantenimiento basado en condición [34]. Estos umbrales se denotan como $d_{1}$ y $d_{2}$, con $d_{2}<d_{1}$ y son valores reales comprendidos en el intervalo ]0,1[, calculados según el método de simulación de costo descrito en la siguiente sección. En la figura 4, se observa de forma gráfica la escala de probabilidad de falla y las acciones por tomar según sea el caso.

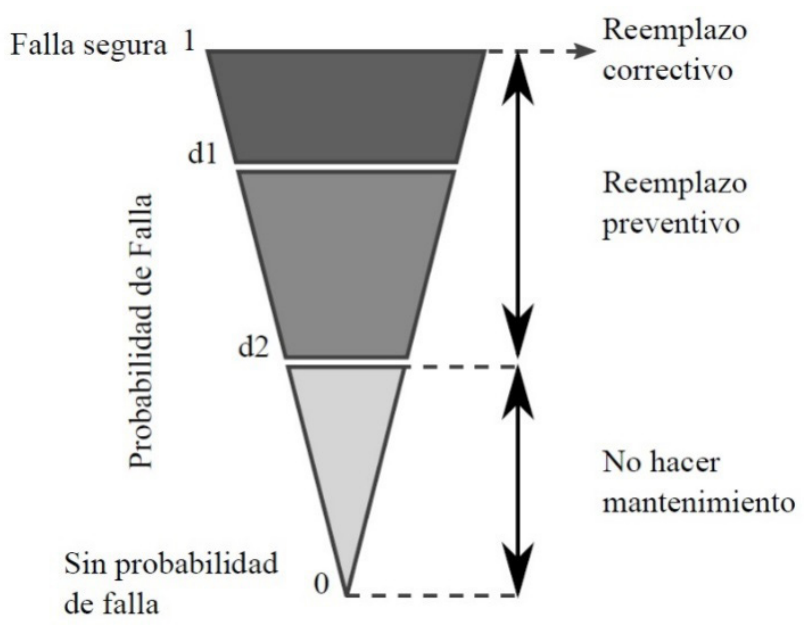

Figura 4. Gráfica para toma de decisiones de mantenimiento según la probabilidad de falla.

Una vez que ambos valores umbrales se especifican, la política de mantenimiento basado en condición queda completamente definida, y la estrategia para sistemas de generación eólica se aplica según lo propuesto por [34], tal y como se describe en los siguientes puntos:

a. Realizar un reemplazo correctivo si un componente falla.

b. Efectuar acciones preventivas en los componentes de una turbina $n$ si $\operatorname{Pr}_{n}>d_{1}$.

c. Si se le debe realizar una intervención preventiva a la turbina $n$, se ejecutan las acciones preventivas a los componentes, tal que la probabilidad de falla de la turbina alcance un valor menor que $d_{2}$. 
Modelo de optimización del mantenimiento basado en la condición

La optimización de la política de mantenimiento basado en la condición consiste en minimizar el costo total esperado de mantenimiento por unidad de tiempo $\left(C_{E}\right)$, encontrando para ello los valores umbrales óptimos $d_{1}$ y $d_{2}$. Según [35], la manera de formular este problema de optimización se muestra en la ecuación 8.

$$
\begin{gathered}
\min \mathrm{C}_{\mathrm{E}}\left(\mathrm{d}_{1}, \mathrm{~d}_{2}\right) \\
\text { Restricción: } 0<d_{2}<d_{1}<1
\end{gathered}
$$

Dada la complejidad del problema de optimización planteado, se recurre a un método de simulación para evaluar el costo, según la estrategia presentada en [34]. El flujograma de la figura 5 contiene el procedimiento para ejecutar la simulación citada.

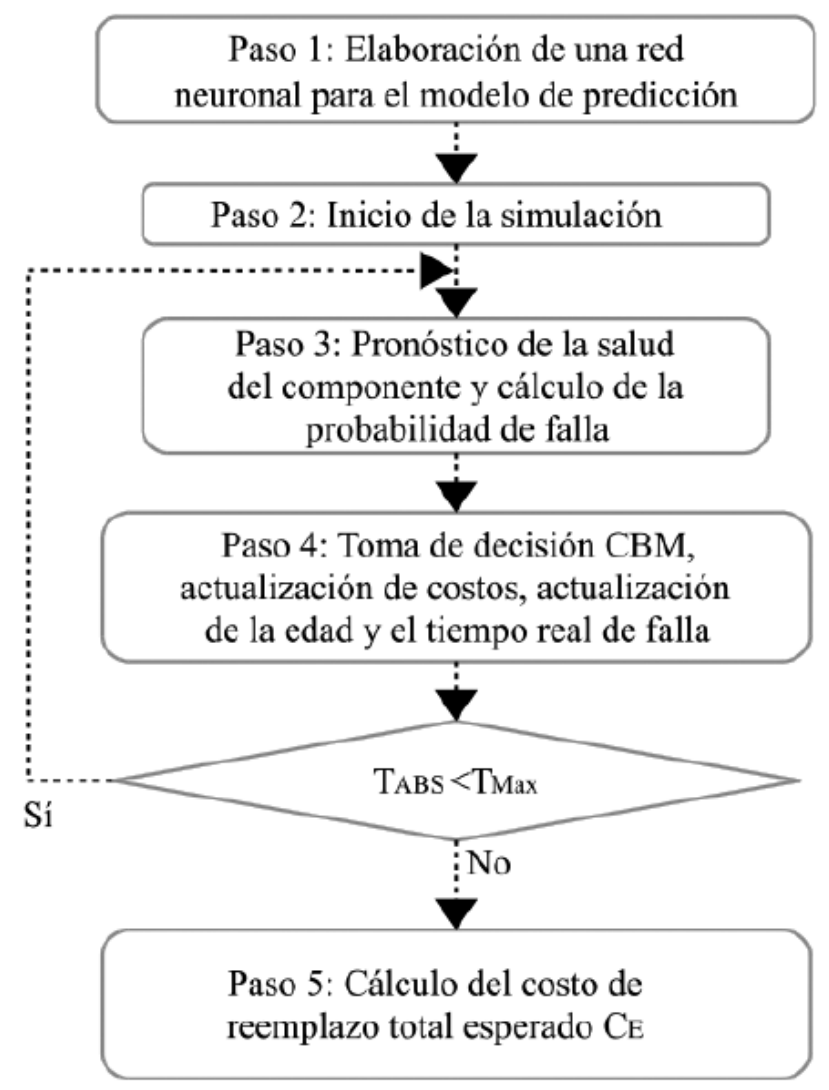

Figura 5. Flujograma para la optimización del costo total esperado de mantenimiento.

Del proceso de entrenamiento de la RNA, se obtienen la media $\left(\mu_{p, m}\right)$ y la desviación estándar $\left(\sigma_{p, m}\right)$ del error de predicción del porcentaje de vida. Posteriormente, se generan tiempos reales de falla para cada componente de cada turbina $\left(T L_{n, m}\right)$, por medio de un muestreo de la distribución Weibull del componente en estudio.

El tiempo de falla previsto $T P_{n, m}$ de los componentes, se genera a partir del muestreo de la distribución normal $N\left(T L_{n, m}, \sigma_{p} \cdot T L_{n, m}\right)$, donde se utiliza $\sigma_{p}$ para incluir el efecto del error en la predicción. Con el TP calculado, la probabilidad actual de falla para un componente se calcula según la ecuación 9. 


$$
P r_{n, m}=\frac{\int_{t_{i}}^{t_{i}+L} \frac{1}{\sigma_{p} T P_{n, m} \sqrt{2 \pi}} e^{-0,5\left(\frac{x-T P_{n, m}}{\sigma_{p} T P_{n, m}}\right)^{2}} d x}{\int_{t_{i}}^{\infty} \frac{1}{\sigma_{p} T P_{n, m} \sqrt{2 \pi}} e^{-0,5\left(\frac{x-T P_{n, m}}{\sigma_{p} T P_{n, m}}\right)^{2}} d x}
$$

Se actualizan el costo, la edad del componente y los valores reales de tiempo de falla, incluyendo los siguientes aspectos:

a. Para todo componente cuya edad iguale o supere $T L_{n, m}$, se incurre en un costo asociado a un reemplazo correctivo.

b. Existirá un costo por reemplazo preventivo de los componentes con mayor riesgo de falla si $\operatorname{Pr}_{n}>d_{1}$, hasta lograr que $\operatorname{Pr}_{n}<d_{2}$.

c. Siempre habrá un costo asociado al envío de personal de mantenimiento al parque eólico, el cual debe ser considerado al efectuar un reemplazo de cualquier tipo.

d. Al reemplazar algún componente, el tiempo se moverá hacia el final del tiempo de logística, y se debe generar un nuevo $T L_{n, m}$. Por otro lado, si no hay reemplazos por hacer, la movilización de tiempo es hacia el próximo tiempo de inspección.

Cuando se finaliza la simulación, se calcula el costo total esperado por unidad de tiempo.

\section{Configuración requerida}

A modo de ejemplo, en la figura 1 se muestra un parque eólico de 25 turbinas eólicas, cada una con los cuatro componentes seleccionados para el estudio.

El hecho de que los componentes se encuentren configurados en serie, significa que la falla en alguno de ellos conducirá a la falla de la turbina. Por otro lado, el parque eólico tiene sus turbinas conectadas en paralelo, lo cual implica que el sistema estará en falla únicamente si todos los aerogeneradores dejan de funcionar.

Datos iniciales

Los datos iniciales necesarios para la implementación del modelo son:

1. Historiales de falla y suspensión: Estos historiales se utilizan para el entrenamiento de las redes neuronales, por lo tanto, la exactitud de cada red será directamente proporcional a la información se tenga.

2. Parámetros de la distribución Weibull del tiempo de vida: Se ha demostrado que la distribución Weibull se ajusta a las conductas de falla de numerosas partes mecánicas y eléctricas [36]. Esta información se encuentra en publicaciones de la industria eólica o por medio de los fabricantes de cada componente.

3. Costos fijos y variables de mantenimiento: Se deben incluir los costos variables por reemplazo correctivo y preventivo para cada componente y los costos fijos y de envío de personal al proyecto eólico.

Construcción de las redes neuronales artificiales

El análisis de vibraciones es una de las técnicas predominantes para el monitoreo de condición [37], por lo cual, se considera como variable de entrada para las RNA del rotor, rodamiento principal y caja multiplicadora. 
La norma ISO 10816-21, establece que deben existir mediciones de vibración en las direcciones axial, vertical y horizontal [38]. La medida recomendada por la norma es la de aceleración (en $\mathrm{m} / \mathrm{s}^{2}$ ), debido a la buena respuesta ante frecuencias altas.

La topología de la RNA para el rotor es idéntica a la del rodamiento principal, con ocho nodos de entrada correspondientes a la edad calendario y mediciones de vibración en los tres ejes que indica la norma, la primera capa oculta con cuatro nodos, la segunda con dos nodos y una unidad neuronal de salida.

Como se mostró, la caja multiplicadora es un componente de especial interés para el modelo. Por la anterior razón, además del análisis de vibraciones, se incluye el monitoreo de aceite en línea, con el fin de obtener un mejor reflejo de su condición [39]. La RNA propuesta para la caja multiplicadora incluye diez nodos de entrada correspondientes a la edad calendario, medición de vibraciones en los tres puntos indicados por la norma y la condición del aceite, cinco nodos en la primera capa oculta, tres nodos en la segunda y la respectiva capa de salida.

Al generador eléctrico se le monitorean parámetros de voltaje y temperatura, puesto que los mismos evidencian la condición de este componente [40]. La RNA propuesta en este caso incluye seis nodos de entrada, pertenecientes a la edad calendario, voltaje y temperatura, la primera capa oculta con tres nodos, la segunda con dos nodos y la capa de salida.

\section{Conclusiones y trabajo a futuro}

Una manera de aumentar la competitividad de la energía eólica, consiste en generar estrategias cuyo enfoque sea la optimización de los costos de operación y mantenimiento, los cuales son los más significativos dentro del proceso de explotación de la energía a partir del viento.

Se demuestra que la recolección de datos históricos de falla aporta información valiosa sobre el comportamiento de los sistemas en su entorno operacional y que debe ser tomada en cuenta para todo plan de mantenimiento, indistintamente de la estrategia a seguir; punto en el cual la industria eólica costarricense presenta un rezago.

Para cada componente, las variables de condición seleccionadas se consideran como representativas de la condición, sin embargo, las mismas son susceptibles a modificarse o eliminarse, mediante el análisis de pruebas experimentales que ayuden a determinar la relevancia de cada variable.

En el presente estudio no se consideran las irregularidades en la carga mecánica generadas por las variaciones en la velocidad del viento, por lo que este debe ser un punto en consideración si se desea aplicar el modelo de mantenimiento propuesto.

Debido a que este estudio se encuentra en su fase inicial, no es posible cuantificar un ahorro esperado, sin embargo, considerando que la mayoría de los aerogeneradores modernos cuentan con sistemas de adquisición de datos, la inversión inicial en cuanto a hardware para aplicar el modelo podría ser relativamente baja.

\section{Agradecimientos}

Se agradece al Dr. Ming J. Zuo, a todo el personal del Laboratorio de Investigación en Confiabilidad (Reliability Research Lab) de la Universidad de Alberta en Canadá y al Instituto Tecnológico de Costa Rica. 


\section{Referencias}

[1] W. Kuo and M. Zuo, Optimal Reliability Modeling: Principles and Applications. John Wiley \& Sons Inc., 2003.

[2] C. Cassady et al., "Selective maintenance modeling for industrial systems", Journal of Quality in Maintenance Engineering, vol. 7, no. 2, pp. 104-117, 2001.

[3] US Environmental Protection Agency. (2010). Deepwater Horizon - BP Gulf of Mexico Oil Spill. [Online]. Available https://www.epa.gov/enforcement/deepwater-horizon-bp-gulf-mexico-oil-spill

[4] E. Byon and Y. Ding, "Season-Dependent Condition-Based Maintenance for a Wind Turbine Using a Partially Observed Markov Decision Process", IEEE Transactions on Power Systems, vol. 25, no. 4, pp. 1823-1834, Nov. 2010.

[5] R.F. Brandao et al., "Neural Networks for Condition Monitoring of Wind Turbines Gearbox", Journal of Energy and Power Engineering, vol. 6, pp. 638-644, Apr. 2012.

[6] J. Acuña, Ingeniería en Confiabilidad. Cartago, Costa Rica: Editorial Tecnológica de Costa Rica, 2003.

[7] M. Soto. (2015, Set. 24). Costa Rica aplaza meta de carbono neutralidad para el año 2100. La Nación. [Online]. Available: http://www.nacion.com/vivir/ambiente/Pais-aplaza-meta-carbono-neutralidad_0_1514048598.html

[8] The Wind Power. Production capacities, Costa Rica. [Online]. Available http://www.thewindpower.net/country_en_29_costa-rica.php

[9] R. Billinton, R. et al., Reliability and Risk Evaluation of Wind Integrated Power Systems. India: Springer, 2013. doi 10.1007/978-81-322-0987-4.

[10] A. Leite et al., "Probabilistic wind farms generation model for reliability studies applied to Brazilian sites", IEEE Transactions on Power Systems, vol. 21, no. 4, pp. 1493-1501, Nov. 2016.

[11] J. Luna, private communication, Mar. 2016.

[12] O. Carvajal, private communication, Apr. 2016.

[13] A. Stenberg and H. Holttinen, "Tuulivoiman tuotantotilastot: Vuosiraportti", Finlandia, 2010.

[14] International Electrotechnical Commission, Wind Turbines, Design Requirements. IEC 61400-1, 2008.

[15] Elforksrapporter. Driftuppföljning av Vindkraftverk Årsrapport. [Online]. Available www.elsforsk.se

[16] J. Ribrant, "Reliability performance and maintenance - A survey of failures in wind power systems," M.S. thesis, KTH School of Electrical Engineering, 2006

[17] S. Sheng and P. Veers, "Wind Turbine Drivetrain Condition Monitoring - An Overview", NREL. NREL/CP-500050698. May 2011.

[18] Centro Nacional de Control de Energía, private communication, Feb. 2016.

[19] Contraloría General de la República. (2015). Serias debilidades en desarrollo y operación del parque eólico Valle Central de la CNFL. [Online]. Available https://cgrfiles.cgr.go.cr/publico/jaguar/Documentos/comunicados/prensa/Boletines/2015/boletin-24-07-2015-eolica.pdf

[20] I. Fornaguera. (2015, Feb. 5). CNFL gasto \$1,6 millones en proyecto eólico entrabado. La Nación. [Online]. Available http://www.nacion.com/nacional/servicios-publicos/CNFL-millones-proyecto-eolicoentrabado_0_1467853218.html

[21] C. Zapata, Confiabilidad en Ingeniería. Colombia: Universidad Tecnológica de Pereira, 2011.

[22] M.K. Pandey, "Selective maintenance for systems under imperfect maintenance policy," Ph.D. dissertation, Mech. Eng. Dept., University of Alberta, Edmonton, 2014.

[23] P.E Labeau and M.C. Segovia, "Effective age models for imperfect maintenance", Proceedings of the Institution of Mechanical Engineers, Part O: Journal of Risk and Reliability, vol. 225, no. 2, pp. 117-130, 2011.

[24] E. Bustamante, El sistema nervioso: desde las neuronas hasta el cerebro humano. Colombia: Editorial Universidad de Antioquia, 2007.

[25] D. Orozco, "Aproximación del uso de redes neuronales en mantenimiento," M.S. thesis. Mech. Eng. Dept., Universidad Eafit, Medellín, Colombia, 2013.

[26] R. Schalkoff, Artificial Neural Networks. McGraw-Hill Education, 1997.

[27] P. Greenwood and L. Wald. Stochastic Neuron Models. Springer International, 2010. 
[28] B. Yegnanarayana, Artificial Neural Networks. India: Prentice Hall, 2006.

[29] S. Marsland, Machine Learning: An algorithmic perspective. CRC Press, 2015.

[30] N, Srivastava, "Dropout: A Simple Way to Prevent Neural Networks from Overfitting", Journal of Machine Learning Research, vol. 15, pp. 1929-1958, 2014.

[31] M. Popescu et al., "Multilayer Perceptron and Neural Networks", WSEAS Transactions on Circuits and Systems, vol. 8, no. 7, pp. 579-588, 2009.

[32] Adnan, M., "Bearing Prognostics using Neural Network under time varying conditions," M.Sc. dissertation, Faculty of Engineering and Computer Science, Concordia University, Montréal, 2010.

[33] M. Orchard. (2010, Sep.) Pronóstico de fallas y estimación en línea de la vida útil remanente de equipos y componentes. [Online]. Available http://www.emb.cl/electroindustria/articulo.mvc?xid=1465\&tip=7

[34] Z. Tian et al., "Condition based maintenance optimization for wind power generation systems under continuous monitoring", Renewable Energy, vol. 36, pp. 1502-1509, 2011.

[35] B. Wu et al., "Condition based maintenance optimization using neural network based health condition prediction", IEEE Transactions on Systems, Man, and Cybernetics. Part A: Systems and Humans, vol. 29, no. 8, pp. 1151-1163, Dec. 2013.

[36] A. Schömig and O. Rose, "On the Suitability of the Weibull Distribution for the Approximation of Machine Failures", Proceedings of the 2003 Industrial Engineering Research Conference, Portland, OR, May 18-20, 2003.

[37] G. White, Introducción al Análisis de Vibraciones. MA: Azima DLI, 2010.

[38] Mechanical vibration - Evaluation of machine vibration by measurements on non-rotating parts - Part 21: Horizontal axis Wind turbines with gearbox. ISO 10816-21, 2015.

[39] J. Terradillos and J. Ciria, "Análisis del aceite como herramienta de mejora del comportamiento de las multiplicadoras de aerogeneradores: Principales problemas detectados a través del lubricante”, IK4-Tekniker, 2013.

[40] M. Castelli and M. Andrade, "Metodología de monitoreo, detección y diagnóstico de fallos en motores asíncronos de inducción", Memorias, no. 6, pp. 9-17, 2008. 